\title{
SURGERY OF THE AORTIC VALVE
}

\author{
Dwight C. McGoon, M.D. \\ Section of Surgery, Mayo Clinic and Mayo Foundation, Rochester, Minnesota
}

ESSENTIALLY all forms of aortic-valve disease can now be repaired or relieved by surgical techniques. In spite of this high degree of attainment, much remains to be accomplished in the evolution of improved techniques and materials and in the evaluation of the adequacy of these methods. The early operative result, the long-standing permanence of the correction, and the durability of prosthetic devices and materials must be scrutinized critically.

The closed approach to the aortic valve has already been tried and discarded. For a period this was the only technique possible, but disease of the aortic valve is not suitable for correction by the closed approach. Even survivors of this treacherous procedure were often little improved, particularly by objective standards. Similarly, although body hypothermia by surface cooling provides 6 to 8 minutes for work on the aortic valve under direct exposure, this is an inadequate period to effect satisfactory relief of acquired, or even congenital, aortic stenosis. Neither of these techniques has provided adequate conditions for the surgeon to attempt repair of the incompetent aortic valve. At present the surgeon must have an hour or more available in which to repair a diseased aortic valve of any type that he may encounter, and adequate time is essential to the unhurried and unharassed atmosphere which is so important. Thus, surgery of the aortic valve now requires extra-corporeal circulation, employing a well-conceived, well-executed, and welltested system of perfusion.

The aortic valve in man is readily accessible through an incision in the root of the aorta. A clamp must be placed across the ascending aorta just proximal to the innominate artery and distal to the aortotomy, while perfusion of the body is maintained by a pump oxygenator. With this system there are no absolute time limitations, although the obvious theoretic objections to long perfusion and extended surgery are pertinent. In certain extenuating circumstances, perfusion has been required for three or more hours and a satisfactory long-term result has been attained.

Obviously, normal perfusion of the coronary arteries is impossible during the period of aortic cross-clamping and exposure of the aortic valve. The myocardial ischæmia which would result therefrom must be controlled by some means. Although ample clinical experience indicates that the myocardium will tolerate ischæmia well for I 5 to 20 minutes, tolerance of ischæmia for longer periods at normothermia, especially in the presence of a greatly hypertrophied ventricle, would be expected to be poor. Two methods of overcoming this problem have received wide clinical application. Cannulas of various kinds have been held or fixed within the coronary ostia through which blood has been pumped to perfuse the coronary system (Littlefield, Lowicki, and Muller, 1960). This is an excellent method of supporting the myocardium, but the cannulas must pass through the operative field, where they hamper exposure of the valve. Action of the heart under these conditions results in further obstruction to adequate operating conditions. The second means of controlling myocardial ischæmia is employment of hypothermia, most commonly consisting of mild body hypothermia associated with profound hypothermia of the heart established by placing sterile ice in the pericardial space (Hufnagel and Conrad, 196r). The chief drawback of this technique is the high incidence of ventricular fibrillation which occurs during re-warming of the myocardium and which cannot be converted to a normal rhythm until re-warming has been completed. Even a relatively minor degree of aortic incompetence then results in considerable loss of the perfusion fluid through the vent placed in the left ventricle, with reduction of blood flow through the remainder of the body during this period.

Need exists for a method of suspending myocardial metabolism which is more effective and reversible than hypothermia.

\section{Congenital Aortic Stenosis}

Before aortic-valve surgery became feasible, congenital aortic stenosis was regarded as a relatively benign condition. This belief was partially founded in fact, since all aortic systolic murmurs do not indicate significant hæmodynamic derangement; it also was fostered by a large element. of 


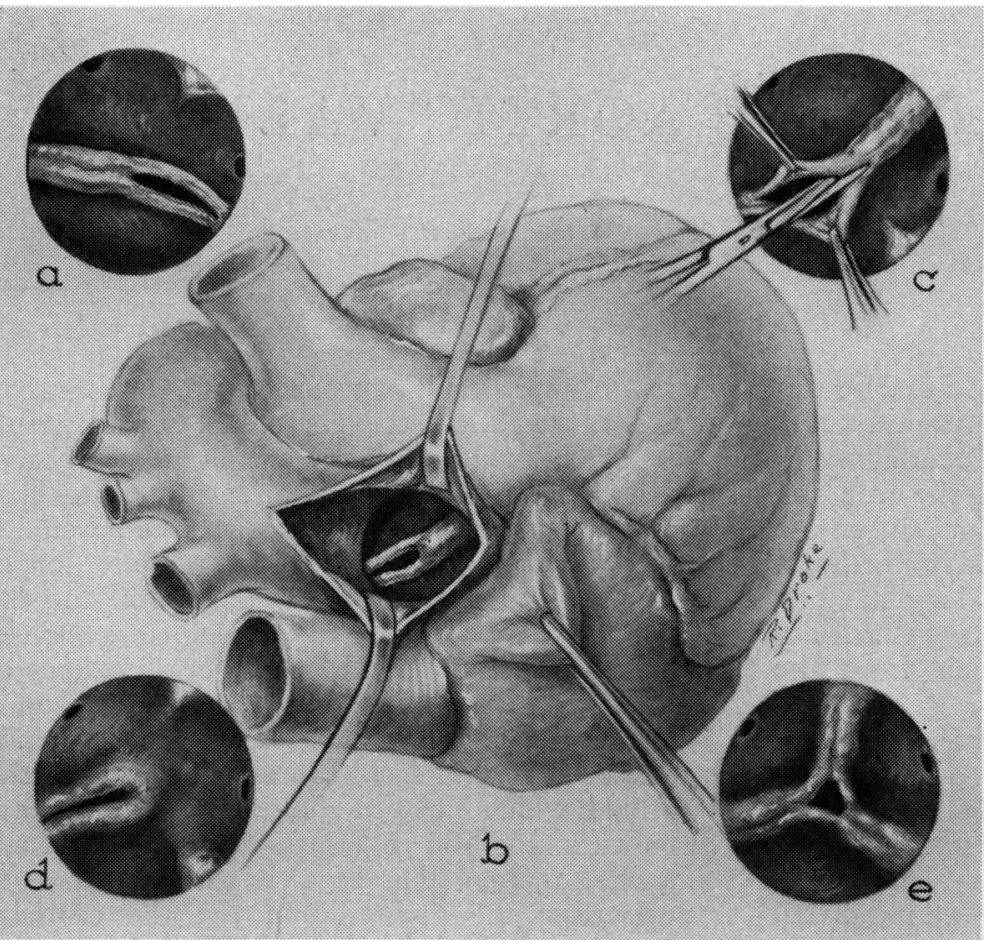

Fig. I. $a$ to $e$.-Exposure of th $\overrightarrow{\vec{a}}$ aortic valve and variations in pathological conditions ers countered at operation fö congenital aortic stenosi (Reprinted with permissio from Ellis, F. H., Jr., and Kirklin, J. W.: Congenit/ Valvular Aortic Stenosis: Anatomic Findings and SuP gical Technic, f. thorac: Surg. in press.)

over-optimism. That heart failure and the classic symptoms of angina, syncope, and dyspnœa, as well as sudden unheralded death, can result from congenital aortic stenosis is now well established. The severe forms of congenital aortic stenosis, therefore, must be selected for surgical treatment.

The classic signs of aortic stenosis include an aortic systolic murmur and thrill with transmission to the carotid artery, soft or absent second aortic sound, and a tendency to a weak peripheral pulse due to the damping effect of the stenosis on the pulse wave. The left ventricle may appear large on the rœntgenogram, but because dilatation of the left ventricle is not present, cardiac size often is not greatly increased. The electrocardiographic signs of left ventricular hypertrophy are nearly always present in significant aortic stenosis, and 'strain' patterns indicate an element of urgency in the need for surgical treatment.

For the individual patient, documentation of the degree of hæmodynamic derangement of the aortic valve is usually needed, since evaluation of the foregoing symptoms and signs of aortic stenosis may not always be a clear indication of its severity. Detailed evaluation of function of the aortic valve requires analysis of the transaortic-valve pressure gradient (Brock and Fleming, 1956). Theoretically, this must be related to the cardiac output, since the pressure drop acrs the stenotic valve is dependent on the rate 0 blood flow through it. However, in the absenc릉 of profound heart failure, general anæsthesio exercise, excitement, or other conditions whic might significantly alter cardiac output, knowledge of the transaortic-valve gradient alone may pro vide enough practical information to permit decisions regarding the need for surgery. Thus a gradient of $75 \mathrm{~mm}$. of mercury or more strongly indicates the need for surgical relief, whereas gradient less than $50 \mathrm{~mm}$. of mercury suggests that the patient should be observed further at. regular intervals. When the transaortic-valve gradient is between 50 and $75 \mathrm{~mm}$. of mercurye the decision for or against operation must be based on individual circumstances.

Congenital stenosis in the aortic-valve area ma occur at three possible sites: just above or down stream from the valve (supravalvular), at the valve (valvular), or below or upstream from the valvg (subvalvular). Pre-operative differentiation of the exact site of congenital aortic stenosis requires that pressures be compared during the witho drawal of a catheter through the zone betweet the left ventricle and the ascending aorta or that selective angiocardiography be performed.

Valvular Aortic Stenosis. - The most commo form is at the valve itself (Fig. I). Such a valve 
may be bicuspid, with fusion to a variable degree of either one or both commissures. Often a raphe bisects one of the leaflets, suggesting that it is a vestige of the undeveloped commissure. The technique of repair then is obvious, namely, incision of the fused commissure. This must be done very precisely, without injury to the free edges of either of the adjacent leaflets.

To complicate the problem, however, the valve ring itself may be found to be small, or the leaflets may be tautly drawn across the orifice, allowing little movement of the leaflets away from the valve centre during systole. Therefore, residual stenosis may be present even after complete opening of the two commissures. Then the temptation to divide one of the leaflets, particularly at the vestigial raphe, may be strong. The possibility of creating aortic insufficiency thereby must be feared, for acceptance of some element of residual stenosis is probably better than adding significant aortic insufficiency. Obviously then, a portion of these patients cannot now be totally relieved of their altered valve function. In a study of the results of surgical treatment for congenital stenosis at the aortic valve, Ellis, Ongley and Kirklin (unpublished data) found evidence of mild or moderate aortic insufficiency in nearly half of their patients, and some element of residual stenosis was present in $60 \%$.

In another form of congenital aortic stenosis, the valve retains a tricuspid architecture but is stenotic by virtue of fusion of one, two, or all three of its commissures. Surgical correction is achieved by incision of each of the fused commissures.

The surgical relief of valvular aortic stenosis is highly safe, carrying an operative risk of about $5 \%$, but the status of the patient, 10,20 , or 30 years hence is highly unpredictable. A remnant of the systolic aortic murmur is virtually always present post-operatively, indicating imperfect streamlining of blood flow through the valve and abnormal vibrations of the leaflets themselves. Such perpetual trauma to the leaflets results in thickening, fibrosis, and finally calcification of the leaflet tissue. Stenosis may recur during middle age because of this, especially in men. Perhaps a significant group of the children treated surgically for valvular aortic stenosis will need reoperation in adult life; this necessity, of course, does not detract from the value or need of the operation earlier in life when the proper indications are present.

Sub-valvular Aortic Stenosis. - The outflow tract of the left ventricle at a level just proximal to the aortic valve is a common site of congenital aortic stenosis: '. In the majority of these instances a

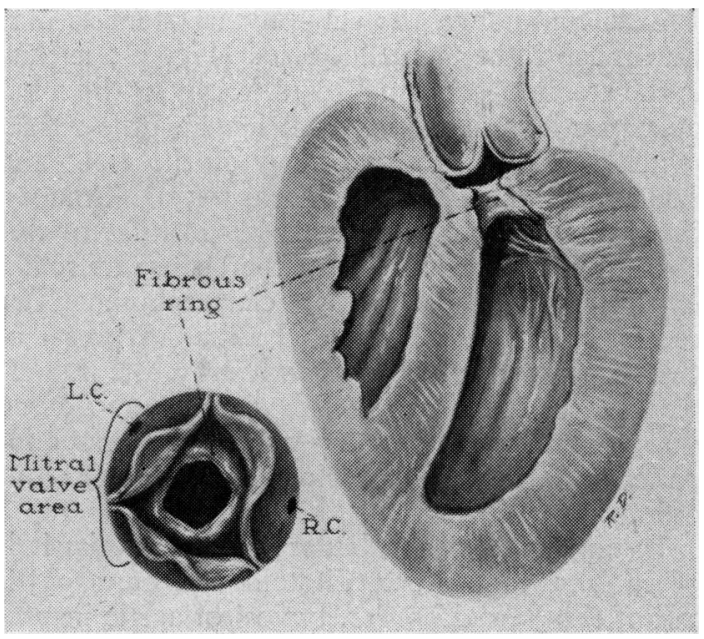

Fig. 2.-Localized subvalvular aortic stenosis. The obstruction, which is partly fibrous, is immediately beneath the base of the leaflets of the aortic valve. (Reprinted with permission from Kirklin, J. W., and Ellis, F. H., Jr.: Surgical Relief of Diffuse Subvalvular Aortic Stenosis, Circulation, in press.)

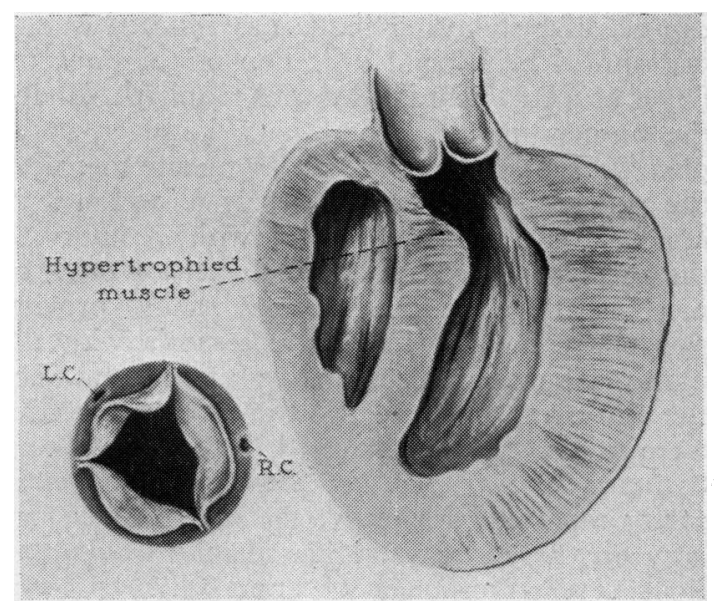

FIG. 3.-Anatomy of diffuse subvalvular aortic stenosis. The obstructing hypertrophied portion of muscle is about $1 \mathrm{~cm}$. upstream from the base of the valve and is considerably more prominent during ventricular systole. For these reasons, at operation, with the heart quiet and the base of the aorta opened, no obstruction is visible in or beneath the aortic valve. (Reprinted with permission from Kirklin, J. W., and Ellis, F. H., Jr.: Surgical Relief of Diffuse Subvalvular Aortic Stenosis, Circulation, in press.)

membrane or diaphragm of fibrous tissue is attached to hypertrophied septal muscle of the outflow tract and to the base of the anterior leaflet of the mitral valve and is often close to the bases of the leaflets themselves (Fig. 2). 
Less commonly, the sub-valvular stenosis may be diffuse rather than localized (Fig. 3); it then is the result of abnormal hypertrophy of the muscular portions of the left ventricular outflow tract which impinge on the lumen of the tract and cause functional stenosis (Brock, I957). Although there may be a congenital predisposition to this condition, the basic cause of the diffuse muscular hypertrophy needs further clarification. The recent report of Kirklin and Ellis (in press) of two successful procedures whereby the hypertrophied muscle in the outflow tract was excised through a left ventriculotomy gives fresh hope for patients having this previously inoperable condition.

In congenital sub-valvular aortic stenosis, it is of interest that a soft diastolic murmur to the left of the sternal border, typical of aortic insufficiency, is usually present in these cases. The basis for this murmur is uncertain, but it probably results from a downward pull on the leaflets of the valve by the sub-valvular membranous diaphragm, preventing their proper co-aptation.

Undue fear for the integrity of the aortic or mitral valve leaflets in the course of excision of sub-valvular stenosing tissue has resulted in the widespread belief that dilatation of this area by finger or instrument is all that is required. Under the proper operative conditions, however, complete excision of the obstructing membrane, with the rim of hypertrophied muscle to which it is attached, can be accomplished without injury to the adjacent leaflets. Thorough excision of all or most of the obstructing tissues should provide this type of congenital aortic stenosis with the most favourable postoperative prognosis, since the aortic-valve leaflets and the coronary arteries were not involved or affected by the lesion.

Supra-valvular Aortic Stenosis. - Although rarely reported previously, the era of open surgery on the aortic valve has already brought to light several instances of supra-valvular aortic stenosis (McGoon, Mankin, Vlad and Kirklin, I96r). The condition is characterized by stenosis of the aorta at the upper limit of the aortic sinuses of Valsalva, in the same transverse plane as the commissural attachments of the aortic valve leaflets to the aorta (Figs. 4 and 5). Thus coarctation exists at the origin of the aorta, and, as with true coarctation of the aorta, the increased thickness of the aortic wall at the site results in a greater luminal narrowing than seems apparent from the outside. A unique feature of this type of stenosis is the fact that the only tributaries of the aorta arising proximal to the site of the stenosis are the right and left coronary arteries.

Kirklin (McGoon and associates, 196r) first successfully relieved this type of aortic stenosis

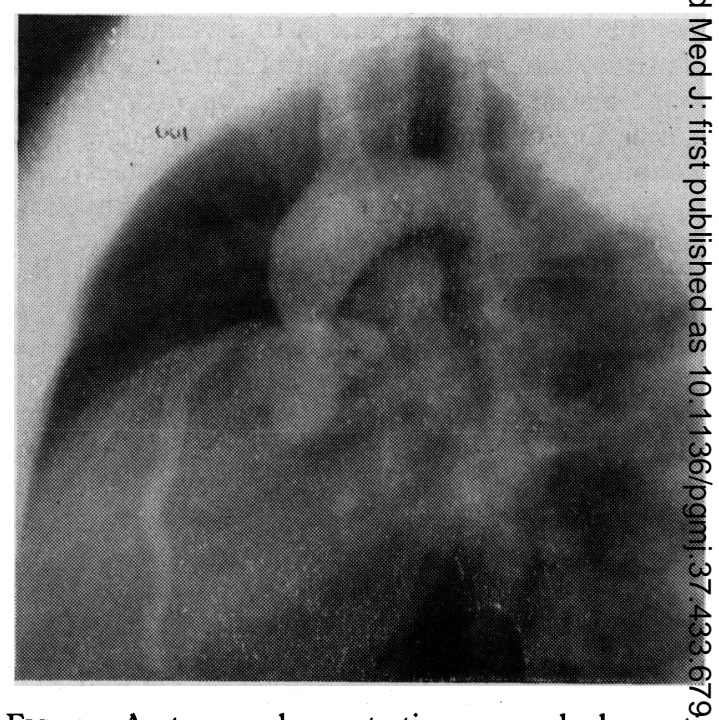

FIG, 4.-Aortogram demonstrating supravalvular aortic stenosis. Origin of coronary arteries is proximal to the stenosis. (Reprinted with permission from McGoon, D. C., Mankin, H. T., Vlad, P., an Kirklin, J. W. (196r): The Surgical Treatment off Supravalvular Aortic Stenosis, f. thorac. Surg, 41, 125.)

by increasing the circumference, and hence $\frac{0}{9} \vec{g}$ diameter, of the stenotic area (Fig. 5). He acconm plished this by suturing a diamond-shaped prosthesis into the lower extent of a long aorte tomy which extended down across the stenot area into the depths of the non-coronary aorto sinus. Operations have now been performed of six such patients at the Mayo Clinic with suet cessful results for all.

Re-catheterization of Kirklin's original patient many months later established the fact that residual gradient was present across the stenotic zone, although the patient had noted some recuㄹ. rence of his angina-like distress. This and study of other hearts indicate the possibility that pre cocious coronary arterial disease may develop this condition from the coronary arterial hype tension prior to relief of the supra-valvulgr stenosis. Careful observation of the other patients operated on will be required to assess the prepensity for coronary artery disease in this type aortic stenosis.

\section{Calcific Aortic Stenosis}

Just as is true of mitral stenosis, rheumatic inflammation is the basis for most instances of acquired calcific aortic stenosis. However, the deformity of the valve may not become hæmødynamically significant until calcification of its leaflets occurs. Calcification of the valve acquired aortic stenosis is so common that 


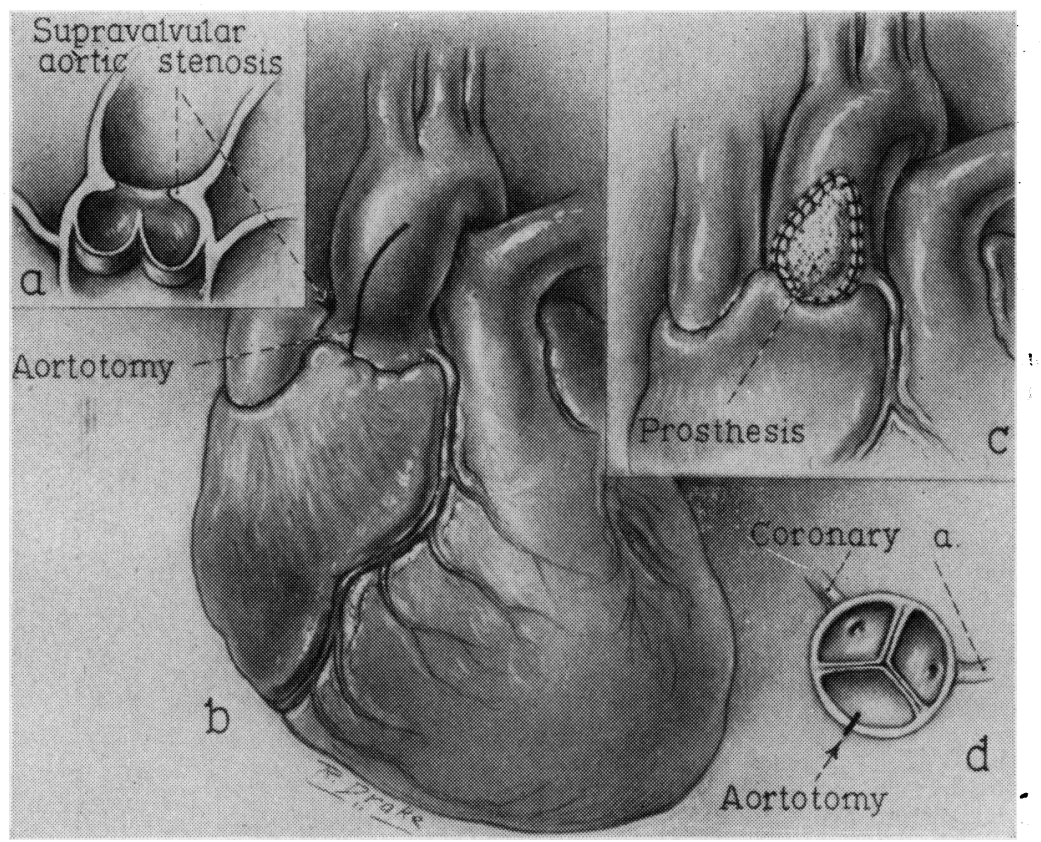

Fig. 5. $a$ to d.-Anatomy of supravalvular aortic stenosis, demonstrating the method of surgical repair. (Reprinted with permission from McGoon, D. C., Mankin, H. T., Vlad, P., and Kirklin, J. W. (1961): The Surgical Treatment of Supravalvular Aortic Stenosis, F. thorac. Surg., 4I, 125.)

reference is made to its presence in the title given this condition, namely, 'calcific aortic stenosis'. However, in some instances calcification may have been superimposed on congenital malformation of the valve. Heavy calcification of the valve often distorts its original architecture, so that establishment of an acquired or congenital basis for the deformity may not be possible, at least from gross inspection at the time of operation.

This disease, as with all degenerative conditions of the cardio-vascular system, affects men much more commonly than women. The stenosis may be rather well tolerated by the otherwise strong heart for an indefinite period of a few years. During this quiet interval when the classic signs of aortic stenosis, as described in the section on congenital stenosis, are present, symptoms may be absent. Then dyspnœa, vertigo, syncope, or angina may appear during exertion or excitement. Interjected into this gradual trend to progressive cardiac disability is the ever-present hazard of unpredictable sudden death, a threat which complicates consideration of the indications for operation. Occasionally a patient who has been placed under observation to await a supposedly opportune time for operation has become suddenly angry; distraught or excited, has been seized with anginal pain, and then has suddenly died. Thus, although many patients with calcific aortic stenosis may survive for several years after the onset of their symptoms, a large group will die within the first 12 months of the appearance of their first symptoms related to cardiac dysfunction.

In practice, then, any patient who has symptoms of aortic stenosis must be considered a possible candidate for operation. Classic physical, electrocardiographic and roentgenographic signs of aortic stenosis, as described previously, plus significant cardiac disability, constitute a sufficient indication for operation. Again, however, preoperative determination of the transaortic-valve pressure gradient, at least, is necessary; this is especially so if there is a hint that coronary arterial disease might be the cause of a major portion of the cardiac disability. Usually at the time of measurement the left ventricular systolic pressure is approximately $225 \mathrm{~mm}$. of mercury when the aortic systolic pressure is approximately I $10 \mathrm{~mm}$., a gradient of $115 \mathrm{~mm}$. Any gradient of more than $75 \mathrm{~mm}$. under basal conditions is highly significant. A gradient of less than $50 \mathrm{~mm}$., as in congenital aortic stenosis, probably does not warrant operative intervention unless rather profound heart failure, indicating low flow across the valve, is present. In the intermediate range between 50 and $75 \mathrm{~mm}$., individual evaluation is necessary. For such border-line situations, a safe and accurate roentgenologic technique for determining the status of the coronary arteries is particularly desirable, since obstructive disease of the coronary arteries may closely simulate the effects of aortic stenosis.

The systolic murmur of aortic stenosis heard over the aortic area is well transmitted to the 


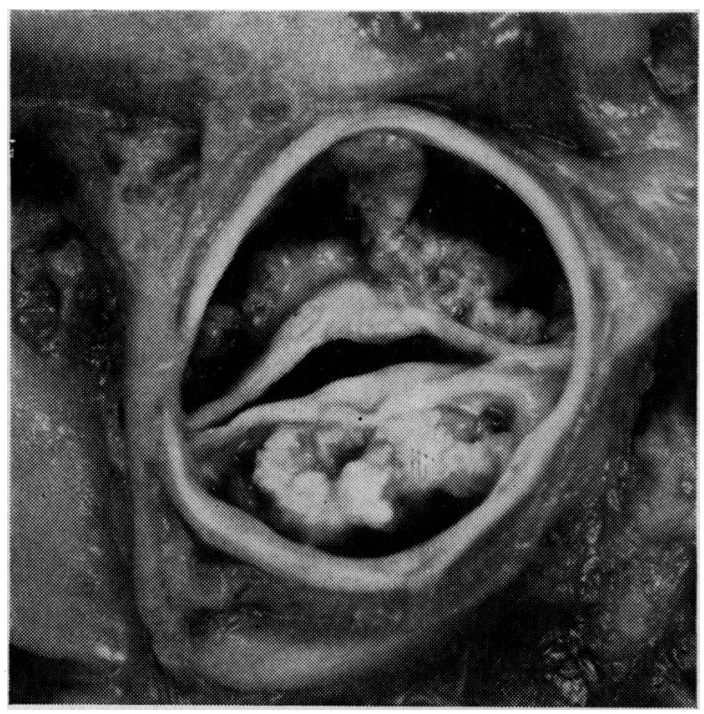

FIG. 6.-Calcified, stenotic aortic valve. The commissure between the right and the left coronary cusps is fused and calcified. Large masses and struts of calcium immobilize the leaflets, but leaflet architecture has been preserved. Mobilization by decalcification of these leaflets should be highly successful.

cardiac apex and often has slightly different auscultatory characteristics at the apex from those at the aortic area. Thus the apical murmur often may cause debate as to the presence or absence of associated mitral insufficiency. Left ventricular failure due to aortic stenosis alone may result in left atrial enlargement and even atrial fibrillation, which further clouds the issue. Special studies at the time of cardiac catheterization, including left atrial pressure curves, dye-dilution curves following injection into the left atrium, left ventricle, and aortic root, and roentgenographic studies following injection of contrast medium into the left ventricle, are helpful in verifying or repudiating the suspicion of associated mitral incompetence.

Commonly a mild degree of aortic insufficiency may be encountered in patients with calcific aortic stenosis, evidenced especially by the classic diminuendo diastolic murmur of the left sternal border. Usually the pulse pressure is widened little, if any, and usually the technique and results of surgery for calcific aortic stenosis are not influenced by this minor degree of aortic incompetence.

A tremendous variation exists in the pathologic aspects encountered in the valve at surgery. The rheumatic residuum has no consistent pattern, and the deposition of calcium is even more haphazard. The calcification usually seems most massive and

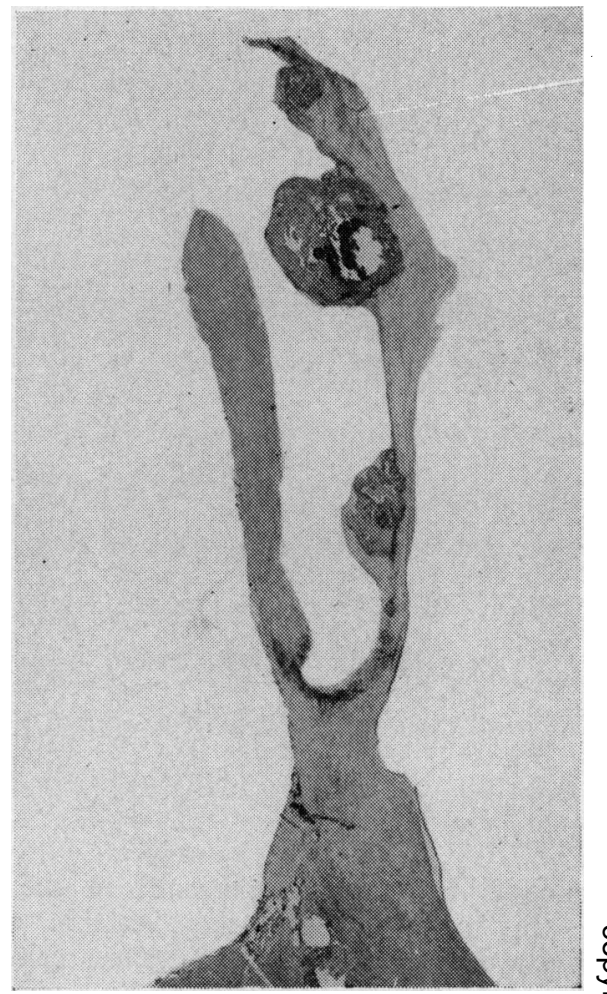

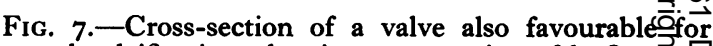
decalcification, showing preservation of leaflet con tinuity in areas of calcium deposition. (Reprinte by permission from Edwards, J. E. (196r): 'Añ Atlas of Acquired Diseases of the Heart and Gregt Vessels'. Philadelphia and London: W. 密 Saunders Co.)

most dense in the region of fused commissures, bridging the zone of fusion and often destroying and replacing all vestiges of the normal leaflet in that area. Leaflets themselves may be replaced by masses of calcium which have eroded through both superior and inferior surfaces of the leaflet. either exposing bare calcium to the blood streang or at best leaving only a thin friable cover of pseudo-endothelium. A more favourable situation for restoration of leaflet mobility is the presence of bars, struts, and masses of rigid calcium (Fig. 6) which seem to involve only one surface or layer of the leaflet as seen in a cross-sections view of that leaflet (Fig. 7). In such an instanced a flexible, intact, movable leaflet may usually boe restored by patient, tedious, delicate teasing of the calcium away from the leaflet in the proper cleavage plane (Bailey, 1958). Mulder and Win field (1960) pointed out that it is technically easier to begin this dissection near the aortio wall at the base of the leaflet, and then to proceed centrally toward its free margin. 
The most common of the possible patterns of calcific aortic stenosis has proved to be a basically tricuspid architecture which has been rendered bicuspid by fusion of the commissure between the right and left coronary leaflets. The other two commissures often remain open.

But whatever the condition encountered, one can usually restore some degree of mobility to the leaflets by tedious piecemeal decalcification and incision or ' fracture' of commissures (Kirklin and Mankin, I960). An unhurried patient atmosphere, fostered by assurance of an adequate system of perfusion and myocardial protection, is essential.

Unfortunately, some portion of nearly all valves, and occasionally nearly all portions of all leaflets, will be beyond restoration, or occasionally efforts to mobilize a leaflet may result in perforation of that leaflet. In such instances, single-leaflet replacement or total-valve replacement must be in the surgeon's armamentarium. Discussion of prostheses for the aortic valve is deferred for the section on aortic insufficiency.

Late results of open repair of calcific aortic stenosis have not been reported in a large group of patients, although a significant number have had these operations. The series at the Mayo Clinic is under study and will be reported in the future in detail. A hospital mortality rate of $15 \%$ or less should now be attainable. The majority of surviving patients have experienced dramatic relief of symptoms, reminiscent of, but not quite equivalent to, the benefits obtained from surgical treatment of mitral stenosis.

The future, with the added experience and length of follow-up, should provide data relative to the incidence and rate of re-calcification and re-stenosis of these valves, and more importantly, should indicate the long-term adequacy of prosthetic reconstruction of the valve. Only then can it be determined whether a conservative restoration of valve mobility is preferable, or whether bold excision and prosthetic reconstruction will assure long-term survival as well as more certain relief of stenosis.

\section{Aortic Insufficiency}

Aortic insufficiency was the last common disease of the cardiac valves to come within the scope of the cardiac surgeon. It is true that some forms of aortic imcompetence were amenable to relief from the moment exposure of the aortic valve was achieved by the technique of extracorporeal circulation; for example, a perforation in a leaflet could merely be patched, or a leaflet cleft by bacterial infection could be sutured (Fig. 8). It was also possible in some instances to restore competence by excision of the noncoronary

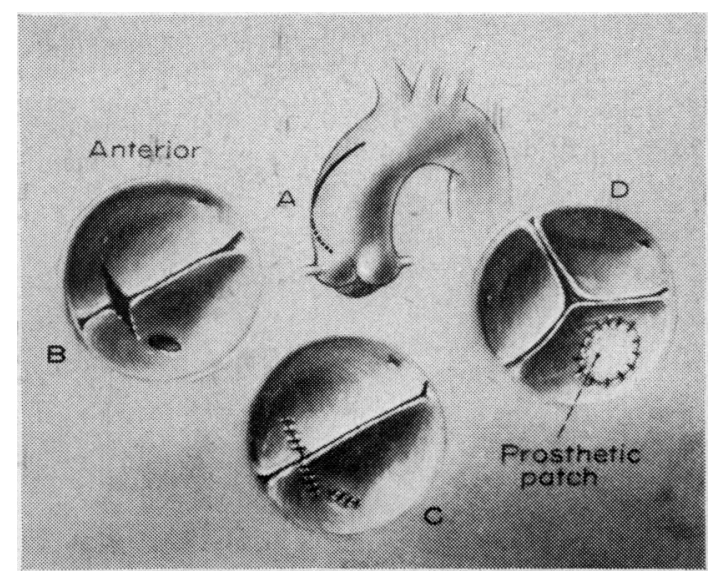

Fig. 8. $A$ to D.-Simple methods of reconstructing a leaflet applicable in relatively few instances. Conditions to which these methods are applicable usually are encountered in patients with postsubacute bacterial endocarditis.

leaflet and the adjacent aor tic sinus and aortic wall, thus creating a bicuspid valve (Garamella and associates, I958; Bailey and Zimmerman, 1959). Also, some degree of competence was thought to be achieved by suturing leaflets together along their adjacent edges (Lillehei, Gott, DeWall and Varco, 1958). But relief of aortic insufficiency could not be undertaken with any confidence until some means of prosthetic repair or reconstruction became feasible.

In addition to the classic physical signs of aortic insufficiency, including the overactive heart, the aortic diastolic murmur, the pounding peripheral pulses, and the roentgenographic and electrocardiagraphic evidence of a huge overactive left ventricle, the diagnosis of aortic insufficiency and the assessment of its severity are greatly aided by the simple determination of arterial blood pressure. Severe aortic incompetence is not present unless the pulse pressure is widened greatly. Thus objective documentation of the function of the valve can be achieved with ease before and after operation.

In most instances, aortic insufficiency results from shortening and deformity of each of the valve leaflets, which involve various dimensions of the leaflets in a variable manner (Fig. 9). Study of the anatomy of the normal valve reveals two striking features: $r$. Length is a definite dimension of the valve, which is probably equally as important to proper function as the cross-sectional area of the valve. 2. Each cusp enters into the formation of a cup-shaped structure which has a somewhat oval top and rounded bottom, one wall of this cup being formed by the corresponding sinus of Valsalva. 


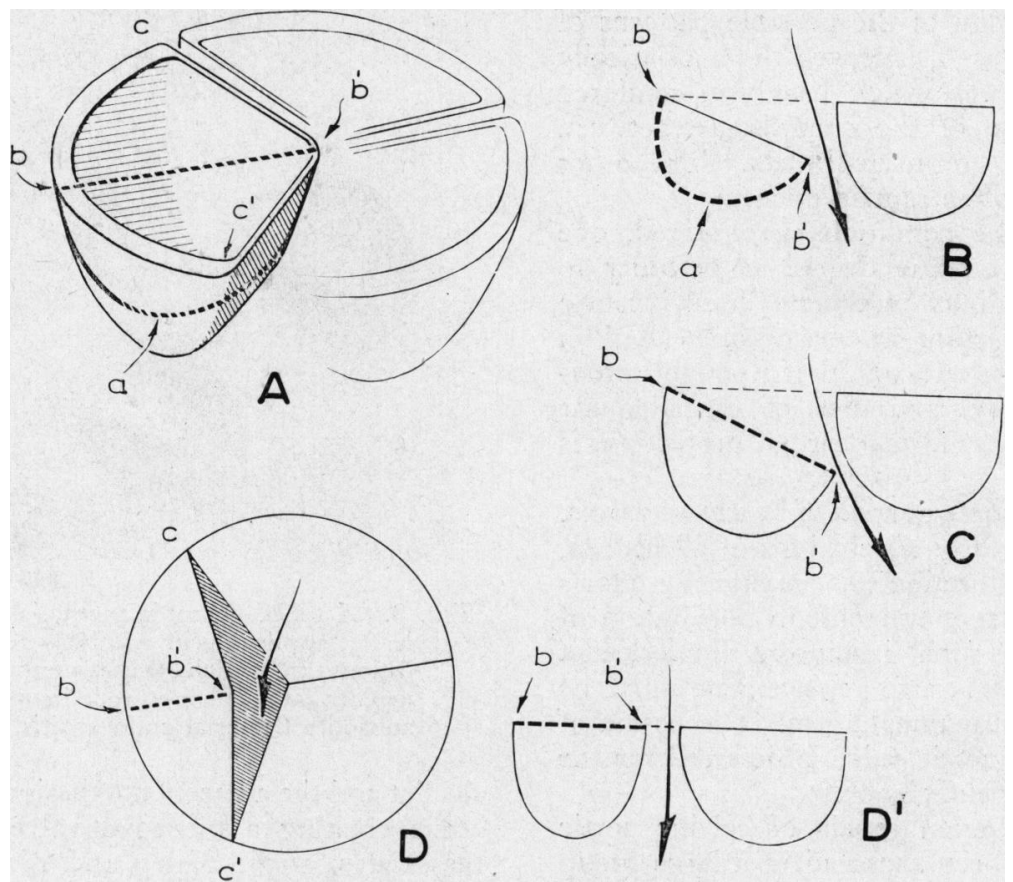

FIG. 9.-Functional anatomy of aortic valve in diastole. $A$. Normal valve. Each cusp or cup is formed by wall of aorta (aortic sinus) and leaflet proper. Length of free margin of leaflet $\left(c\right.$ to $\left.c^{\prime}\right)$ and the distance from the base to the free margin $\left(a\right.$ to $\left.b^{\prime}\right)$ must be correct to allow proper length and spatial arrangement of the critical $b$ to $b^{\prime}$ dimension. $B$. Contraction of leaflet from base to free margin $\left(a\right.$ to $\left.b^{\prime}\right)$ results in downward displacement of free margin of leaflet and failure to coapt with opposing leaflets during diastole. $C$. Lengthening of free margin of leaflet could result in its downward prolapse and hence incompetence of valve. $D$ and $D^{\prime}$. Shortening of free margin of leaflet $\left(c\right.$ to $\left.c^{\prime}\right)$ results in shortening of $b$ to $b^{\prime}$ and prevents closure of valve during diastole. (Reprinted with permission from McGoon, D. C. (196I): Prosthetic Reconstruction of the Aortic Valve, Proc. Staff Meet., Mayo Clin., 36, 75.)

In certain instances where prosthetic reconstruction of the aortic valve is necessary, replacement of a single leaflet may restore competence or relieve stenosis. Hufnagel and Conrad (r96r) and Bahnson, Spencer and Jeckel (196r) have developed prostheses for individual leaflets (Fig. 10). When one leaflet has been damaged, as by bacterial endocarditis, and the remaining leaflets are entirely normal, replacement of a single leaflet is obviously the procedure of choice. But such a situation is rare, and just how satisfactory the replacement of a single leaflet will prove when the remaining leaflets are diseased is not clear.

Since the deformity in aortic insufficiency usually involves all three leaflets of the valve, total replacement with a prosthesis or reconstruction of the valve is commonly required. Most investigators have approached the problem through the development of a pre-constructed valve which they can suture into place at the level of the original aortic valve after excision of all the

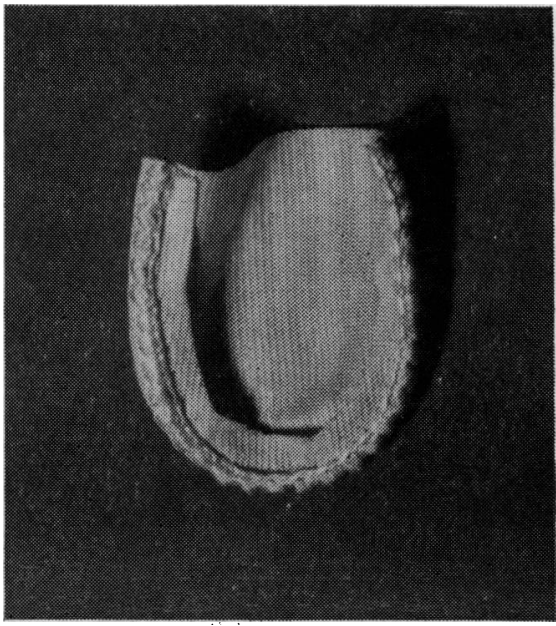

Fig. 10.-Bahnson aortic valve leaflet prosthesis, constructed of knitted
'Teflon. 
leaflet substance. Some success with this method has already been indicated by these workers, including Harken, Saroff, Taylor, Lefemine, Gupta, Lunzer and Low (196r), Hufnagel and Conrad (196r), Kay, Mendelsohn, Nogueira, Suzuki and Zimmerman, (196r), Muller, Littlefield and Dammann, (196r), and Roe and Moore (I96r). Evaluation of the status of the work of each of these investigators is neither possible at present, nor is space available, but certainly the immensity of the task of replacing the aortic valve and the frequency of failure even in the hands of those most familiar with the problem make it necessary to consider prosthetic reconstruction of the aortic valve to be still in its early stage of development.

In most instances of aortic insufficiency, the valve is not totally destroyed or deformed. The major portions of the leaflets remain intact, and the bases of the leaflets are securely and appropriately attached. Shortening and contraction of the leaflets have resulted in merely a central deficiency of valve closure. Therefore, another approach to prosthetic reconstruction of the aortic valve employs the principle that valve function may be restored merely by adding length to the already existing functioning leaflet tissue (McGoon, 196I). According to this scheme, not only is leaflet length restored, but excess leaflet length is added and is properly supported by corresponding lengthening of the commissures. Thus a greater margin of error for leaflet closure is provided than that present even in the normal valve. An appropriate tube of Teflon* may be fashioned at operation to form a total valve prosthesis which fulfils the specific requirements of the particular valve being reconstructed. Increasing clinical and experimental application of this principle suggests that competence of the valve can be restored consistently.

The hospital mortality rate following surgical efforts to relieve advanced severe aortic insufficiency has been high (circa 20 to $45 \%$ ). Therefore, at present deferring operation until the symptoms and disability indicate the approaching loss of all myocardial reserve seems to be in the patient's interest, even though classic physical signs of free aortic incompetence are present. The future, with the added experience and confidence in prosthetic reconstruction which it should provide, will permit earlier operation for aortic incompetence and significant reduction in operative mortality.

\section{Summary}

Essentially all deformities of the aortic valve may now be treated by operation with some confidence by the cardiac surgeon. To do this requires a satisfactory system of whole-body perfusion. Surgical endeavour on the aortic valve is, however, in an early stage of development, so that much improvement in the technique and the result is to be anticipated. Accumulated experience and long-term evaluation of operative results will permit a more enlightened selection of operative techniques for the relief of the various forms of aortic-valve disease.

* Teflon was obtained through the generous cooperation of Mr. Norman Jeckel, U.S. Instrument and Catheter Co., Glens Falls, New York.

\section{REFERENCES}

Bahnson, H. T., Spencer, F. C., and JECKel, N. C. (196r): ' Experiences with Replacement of Individual Cusps of the Aortic Valve'. In Conference on Prosthetic Valves for Cardiac Surgery, pp. 440. Springfield, Illinois: Charles C Thomas.

BAILEY, C. P. (1958): Discussion, F. thorac. Surg., 36, 776.

- , and Zimmerman, J. (1959): The Surgical Correction of Aortic Regurgitation, Amer. F. Cardiol., 3, 6.

Brock, R. (1957): Functional Obstruction. of the Left Ventricle, Guy's Hosp. Rep., I06, 22 I.

, R. C., and Fleming, P. R. (1956): Aortic Subvalvar Stenosis: A Report of 5 Cases Diagnosed During Life, Ibid., ro5, 391 .

Ellis, F. H., JR., ONgley, P. A., and Kirklin, J. W. (unpublished data): Results of Surgical Treatment for Congenital Aortic Stenosis.

Garamella, J. J., Andersen, J. G., and Oropeza, Reuben (1958): The Surgical Treatment of Aortic Insufficiency by Open Plastic Revision of the Tricuspid Aortic Valve to a Bicuspid Valve, Surg., Gynec. Obstet., ro6, 679.

Harken, D. E., Saroff, H. S., Taylor, W. J., Lefemine, A. A., Gupta, S. K., Lunzer, S., and Low, H. B. C. (196r): 'Aortic Valve Replacement'. In Conference on Prosthetic Valves for Cardiac Surgery, pp. 508. Springfield, Illinois: Charles C Thomas.

Hufnagel, C. A., and ConRad, P. W. (196r): 'Prostheses for Aortic Valve Replacement'. In Conference on Prosthetic Valves for Cardiac Surgery, pp. 45I. Springfield, Illinois: Charles C Thomas.

Kay, E. B., Mendelsohn, D., Nogueira, C., Suzuki, A., and Zimmkrman, H. A. (196r): ‘ Surgical Treatment of Aor tic Valvular Disease'. In Conference on Prosthetic Valves for Cardiac Surgery, pp. 483. Springfield, Illinois: Charles C Thomas.

KIrklin, J. W., and Ellis, F. H., JR. (in press): Surgical Relief of Diffuse Subvalvular Aortic Stenosis, Circulation. , and MANKIN, H. T. (1960): Open Operation in the Treatment of Calcific Stenosis, Ibid., $21,578$.

Lillehei, C. W., GotT, V. L., DeWall, R. A., and Varco, R. L. (1958): The Surgical Treatment of Stenotic or Regurgitant Lesions of the Mitral and Aortic Valves by Direct Vision Utilizing a Pump-Oxygenator, $¥$. thorac. Surg., 35, 154 . 
Littlefield, J. B., Lowicki, E. M., and Muller, W. H. J. (i960): Experimental Left Coronary Artery Perfusion Through an Aortotomy During Cardiopulmonary Bypass, Ibid., 40, 685.

McGoon, D. C. (I96I): Prosthetic Reconstruction of the Aortic Valve, Proc. Mayo Clin., 36, 88.

-, Mankin, H. T., VLad, P., and KirkLIN, J. W. (196I): The Surgical Treatment of Supravalvular Aortic Stenơsis, F. thorac. Surg., 41, I25.

Mulder, D. G., and Winfield, M. E. (1960): Valvuloplasty for Acquired Aortic Stenosis, Ann. Surg., 151, 203.

MulleR, W. H., JR., LitTlefield, J. B., and DammanN, J. F., JR. (1961): 'Subcoronary Prosthetic Replacemen?of the Aortic Valve'. In Conference on Prosthetic Valves for Cardiac Surgery, pp. 493. Springfield, Illinois: Cha des C Thomas.

RoE, B. B., and Moore, D. (1961): 'A Satisfactory Flexible Tricuspid Elastomer Prosthetic Valve: Problems of Des. and Implantation'. In Conference on Prosthetic Valves for Cardiac Surgery, pp. 462. Springfield, Illin $\AA_{\circ}$ : Charles C Thomas. 